\title{
THE BEST SOBOLEV TRACE CONSTANT IN DOMAINS WITH HOLES FOR CRITICAL OR SUBCRITICAL EXPONENTS
}

\author{
J. FERNÁNDEZ BONDER ${ }^{1}$, R. ORIVE ${ }^{2}$ and J. D. ROSSI ${ }^{\varpi}$
}

(Received 8 November, 2006)

\begin{abstract}
In this paper we study the best constant in the Sobolev trace embedding $H^{1}(\Omega) \hookrightarrow L^{q}(\partial \Omega)$ in a bounded smooth domain for $1<q \leq 2_{*}=2(N-1) /(N-2)$, that is, critical or subcritical $q$. First, we consider a domain with periodically distributed holes inside which we impose that the involved functions vanish. There exists a critical size of the holes for which the limit problem has an extra term. For sizes larger than critical the best trace constant diverges to infinity and for sizes smaller than critical it converges to the best constant in the domain without holes. Also, we study the problem with the holes located on the boundary of the domain. In this case another critical exists and its extra term appears on the boundary.
\end{abstract}

2000 Mathematics subject classification: primary 35B27, 35J65; secondary 46E35.

Keywords and phrases: homogenization, nonlinear boundary conditions, Sobolev trace embedding.

\section{Introduction}

Sobolev inequalities have been studied by many authors and are by now a classical subject. Their study at least goes back to [2], for more references see [15]. The Sobolev trace inequality is relevant for the study of boundary value problems for differential operators and has been intensively studied, see for example, $[4-6,16]$ and their references. Given a bounded smooth domain $\Omega \subset \mathbb{R}^{N}$, we deal here with the best constant of the Sobolev trace embedding $H^{1}(\Omega) \hookrightarrow L^{q}(\partial \Omega)$ for critical or subcritical

\footnotetext{
'Departamento de Matemática, FCEyN, Universidad de Buenos Aires, Pabellon I, Ciudad Universitaria (1428), Buenos Aires, Argentina; email: jfbonder@dm.uba.ar.

${ }^{2}$ Departamento de Matemáticas, Universidad Autonoma de Madrid Crta., Colmenar Viejo km. 15, 28049 Madrid, Spain; email: rafael.orive@uam.es.

${ }^{3}$ Instituto de Matemáticas y Física Fundamental, Consejo Superior de Investigaciones Científicas, Serrano 123, Madrid, Spain, on leave from Departamento de Matemática, FCEyN UBA (1428), Buenos Aires, Argentina; email: jrossi@dm.uba.ar.

(C) Australian Mathematical Society 2007, Serial-fee code 1446-1811/07
} 
exponents, $1<q \leq 2 *:=2(N-1) /(N-2)$. When $q=2$ this leads to an eigenvalue problem of the Steklov type (see [22]). When $q=2$, this problem is related to the so called Yamabe problem for manifolds with boundaries, see $[1,4,9,16]$.

For subcritical $q, 1<q<2 *$, the embedding is compact and hence there exist extremals [8]. When $q$ is critical, $q=2_{*}$, the embedding is continuous but no longer compact, so the existence of extremals is more involved. In [1] it is proved that if the boundary of the domain contains a point with positive mean curvature then there is an extremal for the embedding. Hence, for any bounded smooth domain there exists an extremal even in the critical case, $q=2$, see also [6].

Homogenization theory was created to model and predict the behaviour of inhomogeneous materials where inhomogeneities take places on a small scale. The homogenization of solutions of boundary-value problems in perforated domains has attracted a lot of attention since the pioneering work of Cioranescu and Murat $[10,12]$. In this paper we consider homogenization problems for the best Sobolev trace constant in perforated domains, following the approach developed in [10] and [12].

First, we consider a domain with holes periodically distributed inside the domain. That is, a bounded smooth domain $\Omega \subset \mathbb{B}^{N}, N \geq 3$, perturbed periodically with holes located at the interior that decrease in size and increase in number as the homogenization parameter $\varepsilon$ goes to zero. We find that there exists a critical size of the holes for which the limit problem has an extra term. For sizes above critical the best trace constant diverges to infinity and for sizes smaller than critical it converges to the best constant in the domain without holes. When we deal with a subcritical exponent we have compactness of the embedding $H^{1}(\Omega) \hookrightarrow L^{q}(\partial \Omega)$, however to prove our result for $q=2$, we need to impose a restriction on the involved domains in order to recover some compactness, in the spirit of [2] (see also [1,6], etc.).

Next, we prove some homogenization results when the semi-holes are located on the boundary of the domain. In this case the critical size of the holes is different from the critical size for holes inside the domain and moreover for the critical size an extra term appears on the boundary.

1.1. Holes in the interior The Sobolev trace constant in domains with a hole (a subdomain of $\Omega$ where the functions are forced to vanish) was first studied in [8] where the authors show that there exists an optimal hole that minimizes the best constant among sets with given volume, in the class of measurable sets. On the other hand, it is also proved that a set $A$ that maximizes $S$ does not exist. In a subsequent paper, [7], the interior regularity of these optimal holes was studied.

For the study of the behaviour of solutions of boundary value problems in domains perturbed periodically with holes complemented with homogeneous boundary conditions (either Dirichlet or Neumann) we refer, for example, to $[10,11,13]$.

Let us first describe the domains that we are considering. Let $B(0, r(\varepsilon)) \subset \mathbb{R}^{N}$ be 
the ball of radius $r(\varepsilon)$, centred at 0 . We assume that $r(\varepsilon)<\varepsilon$ for any $\varepsilon \leq 1$. For each $\varepsilon$ and for any integer vector $n \in \mathbb{Z}^{N}$, we shall denote by $B_{n}^{\varepsilon}$ the translated image of $B(0, r(\varepsilon))$ by the vector $2 n \varepsilon$, that is, $B_{n}^{\varepsilon}=B(0, r(\varepsilon))+2 n \varepsilon$. Also, let us denote by $\mathbf{B}^{\varepsilon}$ the set of all the holes strictly contained in $\Omega$, that is,

$$
\mathbf{B}^{\varepsilon}=\bigcup\left\{B_{n}^{\varepsilon} \mid \operatorname{dist}(2 n \varepsilon, \partial \Omega) \geq \varepsilon, \quad n \in \mathbb{Z}^{N}\right\},
$$

and we set

$$
\Omega^{\varepsilon}=\Omega \backslash \mathbf{B}^{\varepsilon} .
$$

Hence, $\Omega^{\varepsilon}$ is a periodically perforated domain with holes of size $r(\varepsilon)$. All holes have the same shape, the distance between two adjacent holes is of order $\varepsilon$ and they do not overlap. Also, let us remark that the holes are located at a distance of at least $\varepsilon$ from the boundary, $\partial \Omega$. When the holes are allowed to touch the boundary the situation is different; see below and Section 3 . Let us consider the space of functions, $H_{\varepsilon}^{1}(\Omega)=\left\{u \in H^{1}(\Omega):\left.u\right|_{\mathbf{B}^{\mathrm{s}}} \equiv 0\right\}$. The best Sobolev trace constant of the Sobolev embedding $H_{\varepsilon}^{1}(\Omega) \hookrightarrow L^{q}(\partial \Omega)$ is then given by

$$
\lambda(\varepsilon)=\inf _{v \in H_{\varepsilon}^{1}(\Omega) \backslash H_{0}^{1}(\Omega)} \frac{\int_{\Omega}|\nabla v|^{2}+v^{2} d x}{\left(\int_{\partial \Omega}|v|^{q} d S\right)^{2 / q}} .
$$

The extremals, normalized by

$$
\int_{\partial \Omega}|u|^{q} d S=1
$$

are positive in $\Omega^{\varepsilon}$ and weak solutions to

$$
\begin{cases}\Delta u_{\varepsilon}=u_{\varepsilon} & \text { in } \Omega^{\varepsilon}, \\ \frac{\partial u_{\varepsilon}}{\partial \nu}=\lambda(\varepsilon)\left|u_{\varepsilon}\right|^{q-2} u_{\varepsilon} & \text { on } \partial \Omega, \\ u_{\varepsilon}=0 & \text { in } \mathbf{B}^{\varepsilon} .\end{cases}
$$

Our result for interior holes reads:

THEOREM 1.1. Let $\Omega_{\varepsilon}$ be a perforated domain with periodic interior holes of radius $r(\varepsilon)=c_{0} \varepsilon^{a}, c_{0}>0$. Let $q$ be subcritical, $1<q<2_{*}:=2(N-1) /(N-2)$, or critical, $q=2_{*}$. In the critical case we also assume that

$$
\left(\omega_{N}(N-2)\left(c_{0} / 2\right)^{N}+1\right) \frac{|\Omega|}{|\partial \Omega|^{2 / 2 .}}<\frac{1}{K(N)},
$$

where $K(N)$ is given by $K(N):=((N-2) / 2)\left(\omega_{N}\right)^{1 /(N-1)}$ and $\omega_{N}$ is the volume of the unit sphere in $\mathbb{R}^{N}$. Then, 
(1) If $a=N /(N-2)$, then there exists a constant $\mu>0$ (strange term) such that the function $\lambda(\varepsilon)$ converges as $\varepsilon \rightarrow 0$ to $\lambda_{\mu}$ given by

$$
\lambda_{\mu}=\inf _{v \in H^{\prime}(\Omega) \backslash H_{0}^{\prime}(\Omega)} \frac{\int_{\Omega}|\nabla v|^{2}+(1+\mu) v^{2} d x}{\left(\int_{\partial \Omega}|v|^{q} d S\right)^{2 / q}},
$$

with $\mu=\omega_{N}(N-2) c_{0}^{N-2} / 2^{N}$. Moreover, the normalized extremals $u_{\varepsilon}$ converge weakly along subsequences to a normalized extremal of the limit problem (1.5).

(2) If a $>N /(N-2)$, the function $\lambda(\varepsilon)$ converges, as $\varepsilon \rightarrow 0$, to $\lambda_{0}$, the best Sobolev trace constant in the domain without holes, that is,

$$
\lambda_{0}=\inf _{v \in H^{1}(\Omega) \backslash H_{0}^{1}(\Omega)} \frac{\int_{\Omega}|\nabla v|^{2}+v^{2} d x}{\left(\int_{\partial \Omega}|v|^{q} d S\right)^{2 / q}} .
$$

Moreover, the normalized extremals, $u_{\varepsilon}$, converge weakly along subsequences to a normalized extremal of the limit problem (1.6).

(3) If $a<N /(N-2)$, there holds $\lambda(\varepsilon) \rightarrow+\infty$ as $\varepsilon \rightarrow 0$. Moreover, we get a bound for the speed at which $\lambda(\varepsilon)$ goes to $+\infty$,

$$
\lambda(\varepsilon) \leq C \varepsilon^{a(N-2)-N} .
$$

REMARK 1. The constant $K(N)$ is the best Sobolev trace constant in the half-space,

$$
\frac{1}{K(N)}=\inf _{\nabla w \in L^{2}\left(\mathbb{R}_{+}^{N}\right), w \in L^{L_{*}}\left(\partial \mathbb{R}_{+}^{N}\right)} \frac{\int_{\mathbb{R}_{+}^{N}}|\nabla w|^{2} d x}{\left(\int_{\partial \mathbb{R}_{+}^{N}}|w|^{2 \cdot} d x^{\prime}\right)^{2 / 2 \cdot} .}
$$

The constant $K(N)$ is computed in [16].

REMARK 2. From the proof of Theorem 1.1, it can be checked that in the critical case, what is needed is that the best Sobolev trace constants, $\lambda_{\varepsilon}$, for the perforated domains, $\Omega_{\varepsilon}$, be bounded by $1 / K(N)$ uniformly in $\varepsilon$, that is,

$$
\lim _{\varepsilon \rightarrow 0} \lambda(\varepsilon)<\frac{1}{K(N)} .
$$

Condition (1.4) is the simplest condition that assures (1.9).

We note that for every $\varepsilon>0$ it holds that $\lambda(\varepsilon)<1 / K(N)$ for every smooth bounded domain of $\mathbb{R}^{N}$ by [1]. However, from their arguments, it is not obvious that this inequality can be made uniformly strict. 
REMARK 3. The extremals of (1.5) are weak solutions of

$$
\begin{cases}\Delta u=(1+\mu) u & \text { in } \Omega, \\ \frac{\partial u}{\partial \nu}=\lambda_{\mu}|u|^{q-2} u & \text { on } \partial \Omega .\end{cases}
$$

REMARK 4. In the two-dimensional case, that is, $\Omega \subset \mathbb{R}^{2}$, we have an analogous result. The critical radius is now

$$
r(\varepsilon)=\exp \left(-\frac{c_{0}}{\varepsilon^{2}}\right)
$$

Thus, for any radius larger than critical, the best Sobolev constant goes to $\infty$, for any radius smaller than critical it goes to the best constant of the domain without holes and for the critical radius a strange term, $\mu$, appears with $\mu=\pi /\left(2 c_{0}\right)$. The proof of this fact is completely analogous to the case $N \geq 3$ with the choice of an appropriate test function, see $[10,12]$.

REMARK 5. Theorem 1.1 can be generalized to other configuration of the holes. For example, we can consider non-spherical holes, cylinders and trusses, see [10, 12].

1.2. Holes on the boundary We prove some homogenization results when the holes are located on the boundary of the domain. This problem is related to the study of the behaviour of solutions of periodic mixed conditions on the boundary, Dirichlet and Neumann (see for example $[14,20]$ ), and also, with vibration problems of systems with concentrated masses on the boundary (see for example $[18,19]$ ).

To simplify the exposition we consider holes that are placed on a flat part of the boundary. That is, say, $\Omega \subseteq\left\{x_{N} \leq 0\right\}, \Gamma_{1}:=\partial \Omega \cap\left\{x_{N}=0\right\}$ is the closure of a (nonempty) smooth open subset of $\mathbb{R}^{N-1}$. We consider periodically distributed semiholes of size $r(\varepsilon)=c_{0} \varepsilon^{b}$ located only on $\Gamma_{1}$. Assume that $\Gamma_{1}$ is divided by a reticula of size $\varepsilon, \Pi_{\varepsilon}=\left\{(2 m \varepsilon, 0) \in \Gamma_{1} \mid\right.$ with $\left.m \in \mathbb{Z}^{N-1}\right\}$. At each point of the reticula, $x_{i} \in \Pi_{\varepsilon}$, we take a semi-ball of size $r(\varepsilon)$,

$$
S\left(x_{i}, r(\varepsilon)\right)=B\left(x_{i}, r(\varepsilon)\right) \cap\left\{x_{N} \leq 0\right\} \subset \mathbb{R}^{N},
$$

and consider

$$
\mathbf{S}^{\varepsilon}=\bigcup_{x \in \Pi_{\varepsilon}} S\left(x_{i}, r(\varepsilon)\right) \quad \text { such that } S\left(x_{i}, r(\varepsilon)\right) \cap\left(\partial \Omega \backslash \Gamma_{1}\right)=\emptyset,
$$

and $\Omega^{\varepsilon}=\Omega \backslash \mathbf{S}^{\varepsilon}$. Thus, $\Omega^{\varepsilon}$ is a periodically perforated domain with holes of size $r(\varepsilon)$, the distance between two adjacent holes is of order $\varepsilon$ and they do not overlap with $\partial \Omega \backslash \Gamma_{1}$. The holes are located on the boundary but they are "solid" since the semi-balls are considered in $\mathbb{R}^{N}$. We denote $\Gamma_{1}^{\varepsilon}=\Gamma_{1} \cap \mathbf{S}^{\varepsilon}$. As above, we consider the space 
$H_{\varepsilon}^{1}(\Omega)=\left\{u \in H^{1}(\Omega):\left.u\right|_{S^{c}} \equiv 0\right\}$ and the best Sobolev trace constant associated to this space given by (1.1). The extremals normalized by (1.2) are weak solutions of

$$
\begin{cases}\Delta u_{\varepsilon}=u_{\varepsilon} & \text { in } \Omega^{\varepsilon}, \\ \frac{\partial u_{\varepsilon}}{\partial \nu}=\lambda(\varepsilon)\left|u_{\varepsilon}\right|^{q-2} u_{\varepsilon} & \text { on } \partial \Omega \backslash \Gamma_{1}^{\varepsilon}, \\ u_{\varepsilon}=0 & \text { in } \mathbf{S}^{\varepsilon} .\end{cases}
$$

In this case we prove that the critical size is different from the critical size for holes inside the domain and for the critical size an extra term appears on the homogenized part of the boundary, $\Gamma_{1}$.

THEOREM 1.2. Let $q$ be subcritical, $1<q<2$, or critical, $q=2_{*}$. Let $\Omega_{\varepsilon}$ be a perforated domain with periodic boundary holes of radius $r(\varepsilon)=c_{0} \varepsilon^{b}$.

(1) If $b=(N-1) /(N-2)$, the function $\lambda(\varepsilon)$ converges as $\varepsilon \rightarrow 0$ to $\lambda_{\mu_{1}}$, the best Sobolev trace constant in the domain with a weight on the boundary,

$$
\lambda_{\mu_{1}}=\inf _{v \in H^{1}(\Omega) \backslash H_{0}^{1}(\Omega)} \frac{\int_{\Omega}|\nabla v|^{2}+v^{2} d x+\mu_{1} \int_{\Gamma_{1}} v^{2} d S}{\left(\int_{\partial \Omega}|v|^{q} d S\right)^{2 / q}},
$$

where $\mu_{1}=\omega_{N}(N-2) c_{0}^{N-2} / 2^{N}$. Moreover, the normalized extremals $u_{\varepsilon}$ converge weakly along subsequences to a normalized extremal of the limit problem (1.10).

(2) If $b>(N-1) /(N-2)$, the function $\lambda(\varepsilon)$ converges as $\varepsilon \rightarrow 0$ to $\lambda_{0}$, the best Sobolev trace constant in the domain without holes defined in (1.6). Moreover, the normalized extremals $u_{\varepsilon}$ converge weakly along subsequences to a normalized extremal of the limit problem (1.6).

(3) If $b<(N-1) /(N-2)$, the function $\lambda(\varepsilon)$ converges as $\varepsilon \rightarrow 0$ to $\lambda_{1}$, the best Sobolev trace constant in the $\Omega$ among functions that vanish on $\Gamma_{1}$,

$$
\lambda_{0, \Gamma_{1}}=\inf _{v \in H^{1}(\Omega) \backslash H_{0}^{1}(\Omega), v \Gamma_{1} \equiv 0} \frac{\int_{\Omega}|\nabla v|^{2}+v^{2} d x}{\left(\int_{\partial \Omega}|v|^{q} d S\right)^{2 / q}} .
$$

Moreover, the normalized extremals $u_{\varepsilon}$ converge weakly along subsequences to a normalized extremal of the limit problem (1.11).

REMARK 6. For the existence of extremals in the problem of the critical exponent, we need that $\lambda(\varepsilon)$ is uniformly bounded by $1 / K(N)$ with respect to $\varepsilon$. However, under 
our geometric hypothesis, we always have a fixed point on $\partial \Omega-\Gamma_{1}$. (included in the boundary of $\Omega_{\varepsilon}$ ) with positive main curvature and the distance to the holes is uniform (since we place the holes on a flat part of the boundary). So the result of [1] is applied and we have that (1.9) holds. Therefore, in Theorem 1.2, we do not need to impose any extra condition on the domain $\Omega$ for the critical case.

REMARK 7. The extremals of (1.10) are weak solutions to

$$
\begin{cases}\Delta u=u & \text { in } \Omega, \\ \frac{\partial u}{\partial \nu}+\mu_{1} \chi_{\Gamma_{1}} u=\lambda_{\mu_{1}}|u|^{q-2} u & \text { on } \partial \Omega\end{cases}
$$

where $\chi_{\Gamma_{1}}$ is the characteristic function of $\Gamma_{1}$.

REMARK 8. If $\Omega \subset \mathbb{R}^{2}$, we have an analogous result. The critical radius is now

$$
r(\varepsilon)=\exp \left(-\frac{c_{0}}{\varepsilon}\right) \text {. }
$$

Thus, for the critical radius a strange term, $\mu$, appears with $\mu_{1}=\pi /\left(2 c_{0}\right)$.

The rest of the paper is organized as follows: in Section 2 we deal with perforated domains with interior holes and finally in Section 3 we consider holes on the boundary.

\section{Interior holes}

In this section we prove that if we remove from $\Omega$ a periodic set of holes (where we impose that the considered functions vanish) there exists a limit of the best Sobolev trace constant if the size of the holes is not too large. We consider holes which are balls of radius $r(\varepsilon)=c_{0} \varepsilon^{a}$ (see Section 1.1).

First, we construct a sequence of appropriate test functions. Next, we prove Theorem 1.1's distinguishing three cases: $a=N /(N-2), a>N /(N-2)$. and $1 \leq a<N /(N-2)$.

2.1. Construction of $w_{\varepsilon}$ In this subsection, following [10,12], we show that there exists a sequence $w_{\varepsilon}$ that verifies the following assumptions:

(H1) $w_{\varepsilon} \rightarrow 1$ weakly in $H^{1}(\Omega)$.

(H2) $w_{\varepsilon} \equiv 0$ on the holes $B_{n}^{\varepsilon}$.

(H3) There exists a distribution $\mu \in W^{-1, \infty}(\Omega)$ such that for every sequence $v_{\varepsilon}$ that vanishes on the roles and converges weakly in $H^{1}(\Omega)$ to a limit $v$, one has

$$
\left\langle-\Delta w_{\varepsilon}, \varphi v_{\varepsilon}\right\rangle \rightarrow\langle\mu, \varphi v\rangle, \quad \text { for all } \varphi \in C^{\infty}(\bar{\Omega}),
$$

where $\langle$,$\rangle is the duality pairing.$ 
As in $[10,12]$, the function $w_{\varepsilon}$ is defined on each cube $P_{n}^{\varepsilon}=2 n \varepsilon+(-\varepsilon, \varepsilon)^{N}$, $n \in \mathbb{Z}^{N}$, by setting

$$
\begin{cases}w_{\varepsilon}=0 & \text { in } B_{n}^{\varepsilon}, \\ \Delta w_{\varepsilon}=0 & \text { in } T_{n}^{\varepsilon}-B_{n}^{\varepsilon} \\ w_{\varepsilon}=1 & \text { in } P_{n}^{\varepsilon}-T_{n}^{\varepsilon}, \\ w_{\varepsilon} & \text { continuous at the interfaces, }\end{cases}
$$

where $T_{n}^{\varepsilon}=2 n \varepsilon+B(0, \varepsilon)$, with $B(0, \varepsilon)$ the ball with radius $\varepsilon$ and centre 0 . Now it is easy to compute $w_{\varepsilon}$ in polar coordinates in the annulus $T_{n}^{\varepsilon}-B_{n}^{\varepsilon}$. One has

$$
\left.w_{\varepsilon}\right|_{T_{n}^{\varepsilon}-B_{n}^{\varepsilon}}=\frac{r(\varepsilon)^{-(N-2)}-r^{-(N-2)}}{r(\varepsilon)^{-(N-2)}-\varepsilon^{-(N-2)}} \quad \text { if } N \geq 3,
$$

where $r=|x-2 n \varepsilon|$. The result in $[10,12]$ says:

LEMMA 2.1. If we choose $r(\varepsilon)=c_{0} \varepsilon^{N / N-2}$ for $N \geq 3$ with $c_{0}$ a positive constant, then $w_{\varepsilon}$ defined by (2.2) satisfies hypotheses $(\mathrm{H} 1)-(\mathrm{H} 3)$ with

$$
\mu=\frac{\omega_{N}(N-2)}{2^{N}} c_{0}^{N-2},
$$

where $\omega_{N}$ is the surface of the unit sphere in $\mathbb{R}^{N}$.

In order to prove Theorem 1.1, we compute the $L^{2}$-norm of $\nabla w_{\varepsilon}$. As the number $n(\varepsilon)$ of cubes $P_{n}^{\varepsilon}$ with holes is about $|\Omega| /(2 \varepsilon)^{N}$, we get

$$
\left\|\nabla w_{\varepsilon}\right\|_{L^{2}(\Omega)^{N}}^{2} \cong \frac{|\Omega|}{(2 \varepsilon)^{N}} \int_{P_{n}^{\varepsilon}}\left|\nabla w_{\varepsilon}\right|^{2} d x .
$$

Thanks to (2.3) and $r(\varepsilon)<\varepsilon$, we obtain

$$
\left\|\nabla w_{\varepsilon}\right\|_{L^{2}(\Omega)^{N}}^{2} \cong \frac{|\Omega|}{(2 \varepsilon)^{N}} \frac{\omega_{N}(N-2)}{r(\varepsilon)^{-(N-2)}} .
$$

Considering that $r(\varepsilon)=c_{0} \varepsilon^{a}$, we have

$$
\left\|\nabla w_{\varepsilon}\right\|_{L^{2}(\Omega)^{N}}^{2}= \begin{cases}\omega_{N}(N-2)\left(\frac{c_{0}}{2}\right)^{N}|\Omega| \varepsilon^{a(N-2)-N}+o\left(\varepsilon^{a(N-2)-N}\right), & a \neq \frac{N}{N-2}, \\ \omega_{N}(N-2)\left(\frac{c_{0}}{2}\right)^{N}|\Omega|+o(1), & a=\frac{N}{N-2} .\end{cases}
$$

On the other hand, by the definition of $B^{\varepsilon}$, we have that every hole is contained in $\Omega$ and the distance to the boundary is larger than $\varepsilon$. Thus, we get

$$
w_{\varepsilon} \equiv 1 \quad \text { and } \quad \frac{\partial w_{\varepsilon}}{\partial \nu}=0 \quad \text { on } \partial \Omega
$$

Hence,

$$
\int_{\partial \Omega}\left|w_{\varepsilon}\right|^{q} d S=|\partial \Omega| .
$$


2.2. Case $a=N /(N-2)$ Let us observe that, under the assumptions of Theorem 1.1, the best Sobolev trace constants of the perforated domains, $\lambda(\varepsilon)$, are bounded independently of $\varepsilon$. To this end, let us use $w_{\varepsilon}$ as a test function in the infimum that defines $\lambda(\varepsilon)$. We get, using the estimates on $w_{\varepsilon}(2.4)$ and (2.6) proved in the previous subsection,

$$
\lambda(\varepsilon) \leq \frac{\int_{\Omega}\left|\nabla w_{\varepsilon}\right|^{2}+w_{\varepsilon}^{2} d x}{\left(\int_{\partial \Omega}\left|w_{\varepsilon}\right|^{q} d S\right)^{2 / q}} \leq \frac{\left(\omega_{N}(N-2)\left(\frac{c_{0}}{2}\right)^{N}|\Omega|+1\right)|\Omega|}{|\partial \Omega|^{2 / q}}+o(1) .
$$

Hence, the extremals $u_{\varepsilon}$, the weak solutions of (1.3), are bounded in $H^{1}(\Omega)$ and we have, for a subsequence,

$$
u_{\varepsilon} \rightarrow u \geq 0 \quad \text { weakly in } H^{1}(\Omega) .
$$

As the extremals, $u_{\varepsilon}$, are weak solutions of (1.3) and since $w_{\varepsilon}$ vanishes on the holes, $\psi=w_{\varepsilon} \varphi$ with $\varphi \in C^{\infty}(\bar{\Omega})$ is an admissible test function for the weak formulation of (1.3) and satisfies

$$
\int_{\Omega} \nabla \varphi \nabla u_{\varepsilon} w_{\varepsilon} d x+\int_{\Omega} \varphi \nabla u_{\varepsilon} \nabla w_{\varepsilon} d x+\int_{\Omega} u_{\varepsilon} \varphi w_{\varepsilon} d x=\lambda(\varepsilon) \int_{\partial \Omega} u_{\varepsilon}^{q-1} \varphi w_{\varepsilon} d S .
$$

Now we observe that, since $u_{\varepsilon} \rightarrow u, w_{\varepsilon} \rightarrow 1$ strongly in $L^{2}(\partial \Omega)$, we have

$$
\int_{\partial \Omega} u_{\varepsilon}^{q-1} \varphi w_{\varepsilon} d S \rightarrow \int_{\partial \Omega} u^{q-1} \varphi d S .
$$

Similarly, as $u_{\varepsilon} \rightarrow u, w_{\varepsilon} \rightarrow 1$ strongly in $L^{2}(\Omega)$,

$$
\int_{\Omega} u_{\varepsilon} \varphi w_{\varepsilon} d x \rightarrow \int_{\Omega} u \varphi d x
$$

Moreover, since $\nabla u_{\varepsilon} \rightarrow \nabla u$ weakly in $L^{2}(\Omega)$ and $w_{\varepsilon} \rightarrow 1$ strongly in $L^{2}(\Omega)$ we have

$$
\int_{\Omega} \nabla \varphi \nabla u_{\varepsilon} w_{\varepsilon} d x \rightarrow \int_{\Omega} \nabla \varphi \nabla u d x
$$

To deal with the last term, we integrate by parts using that $u_{\varepsilon}$ vanishes on $\mathbf{B}^{\varepsilon}$ and obtain

$$
\int_{\Omega_{\varepsilon}} \varphi \nabla u_{\varepsilon} \nabla w_{\varepsilon} d x=-\int_{\Omega_{\varepsilon}} \nabla \varphi \nabla w_{\varepsilon} u_{\varepsilon} d x-\int_{\Omega_{\varepsilon}} \varphi u_{\varepsilon} \Delta w_{\varepsilon} d x+\int_{\partial \Omega} \varphi u_{\varepsilon} \frac{\partial w_{\varepsilon}}{\partial \nu} d S
$$


We have, by the properties (H1) and (H3) of $w_{\varepsilon}$,

$$
\int_{\Omega} \nabla \varphi \nabla w_{\varepsilon} u_{\varepsilon} d x \rightarrow 0 \quad \text { and } \quad-\int_{\Omega} \varphi u_{\varepsilon} \Delta w_{\varepsilon} d x \rightarrow \int_{\Omega} \mu u \varphi d x
$$

Also, by (2.5),

$$
\int_{\partial \Omega} \varphi u_{\varepsilon} \frac{\partial w_{\varepsilon}}{\partial \nu} d S=0
$$

Now, if we assume that $\lambda(\varepsilon) \rightarrow \lambda$, we have

$$
\int_{\Omega} \nabla \varphi \nabla u d x+\int_{\Omega} \mu u \varphi d x+\int_{\Omega} u \varphi d x=\lambda \int_{\partial \Omega} u^{q-1} \varphi d S .
$$

Let us prove that $\lambda=\lambda_{\mu}$ defined in (1.5). We now distinguish between the subcritical and the critical cases.

Subcritical case, $1<q<2$. In this case, since the immersion $H^{1}(\Omega) \hookrightarrow L^{q}(\partial \Omega)$ is compact and $\left\|u_{\varepsilon}\right\|_{L^{q}(\partial \Omega)}=1$, we have that $\|u\|_{L^{q}(\partial \Omega)}=1$. Hence, taking $\varphi=u$ in (2.9) we get

$$
\lambda=\int_{\Omega}|\nabla u|^{2}+(1+\mu) u^{2} d x \geq \lambda_{\mu} .
$$

Now, to prove $\lambda \leq \lambda_{\mu}$, let $u_{\mu}$ be an extremal of (1.5) and using $u_{\mu} w_{\varepsilon}$ as a test function in (1.1), we get

$$
\lambda(\varepsilon) \leq \frac{\int_{\Omega}\left|\nabla\left(u_{\mu} w_{\varepsilon}\right)\right|^{2}+\left(u_{\mu} w_{\varepsilon}\right)^{2} d x}{\left(\int_{\partial \Omega}\left|u_{\mu} w_{\varepsilon}\right|^{q} d S\right)^{2 / q}} .
$$

By the results of [3] we obtain that $u_{\mu} \in C^{\alpha}(\bar{\Omega})$ and from the maximum principle and Hopf's Lemma we get that $u_{\mu}$ is strictly positive in $\bar{\Omega}$. Therefore the regularity results of [9] are applicable and we obtain that $u_{\mu} \in C^{\infty}(\bar{\Omega})$. Thus, by hypothesis (H1), we get

$$
\int_{\Omega}\left(u_{\mu} w_{\varepsilon}\right)^{2} d x \rightarrow \int_{\Omega} u_{\mu}^{2} d x \quad \text { and } \quad \int_{\partial \Omega}\left|u_{\mu} w_{\varepsilon}\right|^{q} d S \rightarrow \int_{\partial \Omega}\left|u_{\mu}\right|^{q} d S .
$$

On the other hand, we integrate by parts to obtain

$$
\begin{aligned}
\int_{\Omega}\left|\nabla\left(u_{\mu} w_{\varepsilon}\right)\right|^{2} d x= & \int_{\Omega} w_{\varepsilon}^{2}\left|\nabla u_{\mu}\right|^{2} d x+2 \int_{\Omega} u_{\mu} w_{\varepsilon} \nabla u_{\mu} \nabla w_{\varepsilon} d x \\
& -\int_{\Omega} w_{\varepsilon} \operatorname{div}\left(u_{\mu}^{2} \nabla w_{\varepsilon}\right) d x+\int_{\partial \Omega} w_{\varepsilon} u_{\mu}^{2} \frac{\partial w_{\varepsilon}}{\partial \nu} d S \\
= & \int_{\Omega} w_{\varepsilon}^{2}\left|\nabla u_{\mu}\right|^{2} d x-\int_{\Omega} u_{\mu}^{2} w_{\varepsilon} \Delta w_{\varepsilon} d x+\int_{\partial \Omega} u_{\mu}^{2} w_{\varepsilon} \frac{\partial w_{\varepsilon}}{\partial \nu} d S .
\end{aligned}
$$


Thus, by the properties (H1) and (H3) of $w_{\varepsilon}$ and as $u_{\mu} \in C^{\infty}(\bar{\Omega})$, we have

$$
\int_{\Omega} w_{\varepsilon}^{2}\left|\nabla u_{\mu}\right|^{2} d x \rightarrow \int_{\Omega}\left|\nabla u_{\mu}\right|^{2} d x \quad \text { and } \quad-\int_{\Omega} u_{\mu}^{2} w_{\varepsilon} \Delta w_{\varepsilon} d x \rightarrow \int_{\Omega} \mu u_{\mu}^{2} d x .
$$

Also, by (2.5),

$$
\int_{\partial \Omega} u_{\mu}^{2} w_{\varepsilon} \frac{\partial w_{\varepsilon}}{\partial \nu} d S=0
$$

Finally, passing to the limit in (2.10), we get $\lambda \leq \lambda_{\mu}$ and we conclude the proof of the case $a=N /(N-2)$ for subcritical $q$.

Critical case, $q=2$ *. The existence of extremals $u_{\varepsilon}$ for (1.1) was proved in [1] (see also [6]). Let us prove that the weak limit of the extremals verifies $u \neq 0$. To this end we use the following theorem due to [17].

THEOREM 2.2. There exists a constant $B>0$ such that

$$
\left(\int_{\partial \Omega} v^{2 \cdot} d S\right)^{2 / 2 \cdot} \leq K(N) \int_{\Omega}|\nabla v|^{2} d x+B \int_{\Omega} v^{2} d x
$$

for every $v \in H^{1}(\Omega)$. Here $K(N)$ is given by (1.8) and it is sharp.

Now, as $u_{\varepsilon} \geq 0$, it follows that $u \geq 0$ and, by classical regularity theory, $u$ is smooth up to the boundary. By the strong maximum principle and Hopf's lemma, it follows that either 1 i $>0$ or $u \equiv 0$. In order to prove our claim, we have to rule out the possibility of $u \equiv 0$. To do this, we adapt the argument given in [6] (see also [2]) to show that $\|u\|_{L^{2}(\Omega)} \neq 0$. In fact, by Theorem 2.2 , as $u_{\varepsilon}$ are normalized such that $\left\|u_{\varepsilon}\right\|_{L^{2 \cdot}(\partial \Omega)}=1$, we have

$$
1=\left(\int_{\partial \Omega} u_{\varepsilon}^{2 *} d \sigma\right)^{2 / 2 *} \leq K(N) \int_{\Omega}\left|\nabla u_{\varepsilon}\right|^{2} d x+B \int_{\Omega} u_{\varepsilon}^{2} d x
$$

and hence

$$
1 \leq K(N) \lambda(\varepsilon)+(B-K(N)) \int_{\Omega} u_{\varepsilon}^{2} d x .
$$

From the estimate (2.7) and the hypothesis (1.4), we get (1.9). Passing to the limit $\varepsilon \rightarrow 0$ in (2.11) we arrive by (1.9) to

$$
(B-K(N)) \int_{\Omega} u^{2} d x>0
$$

and the claim follows. 
As before, the limit of the extremals $u_{\varepsilon}$, weak solutions of (1.3), satisfies (we assume, as before, that $\lambda(\varepsilon) \rightarrow \lambda$ )

$$
\int_{\Omega} \nabla \varphi \nabla u d x+\int_{\Omega} \mu u \varphi d x+\int_{\Omega} u \varphi d x=\lambda \int_{\partial \Omega} u^{2 *-1} \varphi d S
$$

Taking $\varphi=u$ in (2.12), we arrive at

$$
\int_{\Omega}|\nabla u|^{2}+(1+\mu) u^{2} d x=\lambda \int_{\partial \Omega} u^{2 *} d S
$$

As $u \neq 0$, we have that $\lambda>0$ and $\|u\|_{L^{2 \cdot}(\partial \Omega)} \neq 0$. Therefore

$$
\lambda_{\mu} \leq \frac{\int_{\Omega}|\nabla u|^{2}+(1+\mu) u^{2} d x}{\left(\int_{\partial \Omega} u^{2 *} d S\right)^{2 / 2 *}}=\lambda\left(\int_{\partial \Omega} u^{2 *} d S\right)^{1 /(N-1)} \leq \lambda .
$$

The reverse inequality follows exactly as in the subcritical case.

2.3. Case $a>N /(N-2)$ Again, using as test functions $w^{\varepsilon}$, we get that the best Sobolev trace constants of the perforated domains, $\lambda(\varepsilon)$, are bounded independently of $\varepsilon$. In fact, by (2.4) and (2.6) we obtain

$$
\lambda(\varepsilon) \leq \frac{\left(\left(\frac{c_{0}}{2}\right)^{N} \varepsilon^{a(N-2)-N}+1\right)|\Omega|}{|\partial \Omega|^{2 / q}}+o\left(\varepsilon^{a(N-2)-N}\right) \leq \frac{2|\Omega|}{|\partial \Omega|^{2 / q}}+1 .
$$

Thus, the extremals $u_{\varepsilon}$ are weak solutions of (1.3) and are bounded in $H^{1}(\Omega)$. We consider a subsequence such that $\lambda(\varepsilon) \rightarrow \lambda$ and $u_{\varepsilon} \rightarrow u$ weakly in $H^{1}(\Omega)$.

As in the previous subsection, let us take $w_{\varepsilon} \varphi$ with $\varphi \in C^{\infty}(\bar{\Omega})$ as a test function and we get the weak formulation (2.8). We pass to the limit in this weak formulation and obtain

$$
\int_{\Omega} \nabla \varphi \nabla u d x+\int_{\Omega} u \varphi d x=\lambda \int_{\partial \Omega}|u|^{q-2} u \varphi d S,
$$

since $w_{\varepsilon}$ converges strongly to 1 in $H^{1}(\Omega)$ thanks to $(2.4)$ in the case $a>N /(N-2)$.

Now, as in the previous subsection, we divide the proof according to whether the exponent $q$ is subcritical or critical.

Subcritical case, $1<q<2$. In this case, as $\left\|u_{\varepsilon}\right\|_{L^{q}(\partial \Omega)}=1$ and as the embedding $H^{1}(\Omega) \hookrightarrow L^{q}(\partial \Omega)$ is compact, we conclude that $\|u\|_{L^{q}(\partial \Omega)}=1$. Hence, taking $\varphi=u$ in (2.13), we get that $\lambda \geq \lambda_{0}$ by the definition of $\lambda_{0}$ in (1.6).

To conclude the proof in this case let us prove that $\lambda \leq \lambda_{0}$. Let $u_{0}$ be an extremal of (1.6) and let us use $u_{0} w_{\varepsilon}$ as a test function in (1.1). Thus,

$$
\int_{\Omega}\left|\nabla\left(u_{0} w_{\varepsilon}\right)\right|^{2} d x=\int_{\Omega} u_{0}^{2}\left|\nabla w_{\varepsilon}\right|^{2} d x+\int_{\Omega} w_{\varepsilon}^{2}\left|\nabla u_{0}\right|^{2} d x+2 \int_{\Omega} u_{0} w_{\varepsilon} \nabla u_{0} \nabla w_{\varepsilon} d x .
$$


As $u_{0} \in C^{\infty}(\bar{\Omega})$ (see [9]), and $\nabla w_{\varepsilon} \rightarrow 0$ strongly in $L^{2}(\Omega)$ (by (2.4)), we get

$$
\int_{\Omega}\left|\nabla\left(u_{0} w_{\varepsilon}\right)\right|^{2} d x \rightarrow \int_{\Omega}\left|\nabla u_{0}\right|^{2} d x .
$$

Now, we pass to the limit in (2.10) to obtain

$$
\lambda \leq \frac{\int_{\Omega}\left|\nabla u_{0}\right|^{2}+u_{0}^{2} d x}{\left(\int_{\partial \Omega}\left|u_{0}\right|^{q} d S\right)^{2 / q}}=\lambda_{0} .
$$

This finishes the proof.

Critical case, $q=2_{*}$. In this case, we need to check that $u \neq 0$, but this follows as in the previous subsection. In fact, by Theorem 2.2 we have

$$
\begin{aligned}
1 & =\left(\int_{\partial \Omega}\left|u_{\varepsilon}\right|^{2 *} d S\right)^{2 / 2} \leq K(N) \int_{\Omega}\left|\nabla u_{\varepsilon}\right|^{2} d x+B \int_{\Omega}\left|u_{\varepsilon}\right|^{2} d x \\
& =K(N) \lambda(\varepsilon)+(B-K(N)) \int_{\Omega}\left|u_{\varepsilon}\right|^{2} d x \\
& \rightarrow K(N) \lambda+(B-K(N)) \int_{\Omega}|u|^{2} d x
\end{aligned}
$$

and, as from (1.9) it holds that $K(N) \lambda<1$, the claim follows.

Finally, arguing exactly as before, we conclude that $\lambda=\lambda_{0}$ and that $u$ is an extremal for $\lambda_{0}$. This finishes the proof.

2.4. Case $1 \leq a<N /(N-2)$ In this case we have to prove $\lambda(\varepsilon) \rightarrow \infty$ as $\varepsilon \rightarrow 0$. Suppose, contrary to our claim, that there exists a sequence of $\varepsilon \rightarrow 0$ such that $\lambda(\varepsilon) \leq C$. Then, there exists a sequence of normalized functions $\left\{u_{\varepsilon}\right\}$ in the space $H_{\varepsilon}^{l}(\Omega)$ and satisfying

$$
\int_{\Omega}\left|\nabla u_{\varepsilon}\right|^{2}+u_{\varepsilon}^{2} d x \leq C .
$$

Considering $n(\varepsilon)$, the number of cubes with holes $P_{n}^{\varepsilon}$ contained in $\Omega$, we get

$$
\int_{\Omega}\left|\nabla u_{\varepsilon}\right|^{2} d x \geq \sum_{i=1}^{n(\varepsilon)} \int_{P_{i}^{\varepsilon}}\left|\nabla u_{\varepsilon}\right|^{2} d x .
$$

Let $\lambda_{1}^{\varepsilon}$ be the Poincaré constant of the Sobolev space

$$
H:=\left\{u \in H^{1}(B(0, \varepsilon)-B(0, r(\varepsilon))) \mid u=0 \text { on } \partial B(0, r(\varepsilon))\right\}
$$

It is shown in [21] that

$$
\lambda_{1}^{\varepsilon} \geq C \frac{r(\varepsilon)^{N-2}}{\varepsilon^{N}} \quad \text { for } N \geq 3
$$


Thus, we obtain

$$
\sum_{i=1}^{n(\varepsilon)} \int_{P_{i}^{\varepsilon}}\left|\nabla u_{\varepsilon}\right|^{2} d x \geq C \frac{r(\varepsilon)^{N-2}}{\varepsilon^{N}} \sum_{i=1}^{n(\varepsilon)} \int_{P_{i}^{\varepsilon}}\left|u_{\varepsilon}\right|^{2} d x
$$

and, since $r(\varepsilon)=\varepsilon^{a}$ with $1 \leq a<N /(N-2)$, we obtain by (2.14) and passing to the limit

$$
\lim _{\varepsilon \rightarrow 0} \sum_{i=1}^{n(\varepsilon)} \int_{P_{i}^{\varepsilon}}\left|u_{\varepsilon}\right|^{2} d x=0
$$

Therefore, $u_{\varepsilon}$ converges to 0 in $L^{2}(\Omega)$. This contradicts the normalization condition (1.2) of the sequence $u_{\varepsilon}$. Hence we obtain that $\lambda(\varepsilon) \rightarrow \infty$.

On the other hand, using $w_{\varepsilon}$ as a test function in (1.1) we get, using (2.4), that $w_{\varepsilon} \rightarrow w$ in $L^{2}(\Omega)$ and $(2.6)$,

$$
\lambda(\varepsilon) \leq \frac{\int_{\Omega}\left|\nabla w_{\varepsilon}\right|^{2}+w_{\varepsilon}^{2} d x}{\left(\int_{\partial \Omega}\left|w_{\varepsilon}\right|^{q} d S\right)^{2 / q}} \leq C \varepsilon^{a(N-2)-N}
$$

Thus, with these estimates we conclude (1.7).

\section{Holes on the boundary}

In this section we consider holes on the boundary. Recall that we assume that we are dealing with holes on a flat part of the boundary. We distinguish three cases: $b>(N-1) /(N-2), b=(N-1) /(N-2)$ and $1 \leq b<(N-1) /(N-2)$. The proof of the critical exponent $2_{*}$ is the same as that in the case of interior holes. Here, with our geometric hypothesis of the domain, (1.9) holds (see Remark 6).

3.1. Case $b>(N-1) /(N-2)$ Using the same test function $w_{\varepsilon}$ extended by $w_{\varepsilon} \equiv 1$ to the whole $\Omega$ we have

$$
\left\|\nabla w_{\varepsilon}\right\|_{L^{2}(\Omega)^{N}} \leq \frac{C r(\varepsilon)^{N-2}}{\varepsilon^{N-1}}=C \varepsilon^{b(N-2)-(N-1)} \rightarrow 0 .
$$

Hence $w_{\varepsilon} \rightarrow 1$ strongly in $H^{\prime}(\Omega)$. Using $w_{\varepsilon}$ as a test function in the definition of $\lambda_{1}(\varepsilon)$ we obtain that there exists $C$ independent of $\varepsilon$ such that $\lambda_{1}(\varepsilon) \leq C$. From this point the proof follows exactly the same lines as the case $a>N /(N-2)$ in Section 2.3. However, since the holes are located on the boundary of the domain, 
(2.5) and (2.6) are not satisfied. We know that $w_{\varepsilon}$ converges to 1 in $L^{2}(\partial \Omega)$. Now, we show that

$$
\frac{\partial w_{\varepsilon}}{\partial \nu}=0 \quad \text { on } \partial \Omega
$$

This clearly holds on $\partial \Omega \backslash \Gamma_{1}$ by the definition of $\omega_{\varepsilon}$. Now, for any $\varphi \in L^{2}\left(\Gamma_{1}\right)$, we have

$$
\int_{\Gamma_{1}} \varphi \frac{\partial w_{\varepsilon}}{\partial \nu} d S=\sum_{n=1}^{m(\varepsilon)} \int_{\left(T_{n}^{t}-B_{n}^{\varepsilon}\right) \cap \Gamma_{!}} \varphi \frac{\partial w_{\varepsilon}}{\partial \nu} d S
$$

where $m(\varepsilon) \sim \varepsilon^{1-N}$ is the number of holes placed on $\Gamma_{1}$. Let us consider a single cell such that we may assume that the centre of the cell is centred at $x=0$. Since $\Gamma_{1}$ is considered flat, the normal unit vector is $-e_{N}=(0, \ldots, 0,-1)$. Therefore, when $w_{\varepsilon}$ is considered, we get $\left\langle-e_{N}, \nabla w_{\varepsilon}\right\rangle=0$, and conclude (3.1).

3.2. Case $b=(N-1) /(N-2) \quad$ As in the case of holes in the interior of $\Omega$, the strange term comes from the term that involves $\Delta w_{\varepsilon}$.

We have that $\Delta w_{\varepsilon}$ vanishes except on the spheres $\partial T_{i}^{\varepsilon}$ and $\partial B_{i}^{\varepsilon}$. From the explicit form of $w_{\varepsilon}$ we obtain

$$
-\Delta w_{\varepsilon}=\mu_{\varepsilon}-\gamma_{\varepsilon}
$$

The function $\gamma_{\varepsilon}$ is supported on the spherical boundary of the semi-ball $S_{i}^{\varepsilon}$. We observe that, since $u_{\varepsilon}=0$ on this region,

$$
\left\langle\gamma_{\varepsilon}, u_{\varepsilon}\right\rangle=0 .
$$

For the other side, we know by $[10,12]$ that in this case

$$
\mu_{\varepsilon}=\left.\sum_{i=1}^{m(\varepsilon)} \frac{\partial w_{\varepsilon}}{\partial n}\right|_{\partial T_{i}^{i}} \delta_{i}^{\varepsilon}=\frac{(N-2) c_{0}^{N-2}}{1-c_{0}^{N-2} \varepsilon} \sum_{i=1}^{m(\varepsilon)} \delta_{i}^{\varepsilon}, \quad \text { in } \mathbb{R}^{N},
$$

where $\delta_{i}^{\varepsilon}$ are the Dirac masses supported by $\partial T_{i}^{\varepsilon}$ for $i=1, \ldots, m(\varepsilon)$. Hence, we have

$$
\int_{\Omega} \varphi u_{\varepsilon} \Delta w_{\varepsilon} d x=\sum_{i=1}^{m(\varepsilon)} \int_{\partial T_{i} \cap \Omega} \frac{(N-2) c_{0}^{N-2}}{1-c_{0}^{N-2} \varepsilon} \varphi u_{\varepsilon} d S .
$$

Thanks to the strong convergence of $u_{\varepsilon}$ to $u$ we can pass to the limit and obtain it since as in $[10,12]$

$$
\lim _{\varepsilon \rightarrow 0} \int_{\Omega} \varphi u_{\varepsilon} \Delta w_{\varepsilon} d x=\frac{\omega_{N}(N-2) c_{0}^{N-2}}{2^{N}} \int_{\Gamma_{1}} \varphi u d S=\mu_{1} \int_{\Gamma_{1}} \varphi u d S .
$$

This case is analogous to that of spherical holes periodically distributed on a hyperplane of $\mathbb{R}^{N}$ of $[10,12]$. We note that the capacity of a semi-sphere is a half of the one for the sphere. The rest of the terms can be handled as in the previous section. 
3.3. Case $1 \leq b<(N-1) /(N-2)$ Assume that there exists a sequence $\varepsilon \rightarrow 0$ with normalized extremals $\left\{u_{\varepsilon}\right\}$ in the space $H_{\varepsilon}^{1}(\Omega)$. By the definitions of $\lambda(\varepsilon)$ and $\lambda_{0, \Gamma_{1}}$, we have $\lambda(\varepsilon) \leq \lambda_{0, \Gamma_{1}}$. Therefore

$$
\limsup _{\varepsilon \rightarrow 0} \lambda(\varepsilon) \leq \lambda_{0, \Gamma_{1}}
$$

and the extremals satisfy

$$
\int_{\Omega}\left|\nabla u_{\varepsilon}\right|^{2}+u_{\varepsilon}^{2} d x \leq C .
$$

Considering $m(\varepsilon) \sim \varepsilon^{1-N}$, the number of cells $P_{i}^{\varepsilon}$ placed on $\Gamma_{1}$, we get

$$
\int_{\Omega}\left|\nabla u_{\varepsilon}\right|^{2} d x \geq \sum_{i=1}^{m(\varepsilon)} \int_{P_{i}^{i} \cap \Omega}\left|\nabla u_{\varepsilon}\right|^{2} d x \geq C \frac{\varepsilon^{b(N-2)}}{\varepsilon^{N}} \sum_{i=1}^{m(\varepsilon)} \int_{P_{i}^{i} \cap \Omega}\left|u_{\varepsilon}\right|^{2} d x,
$$

by the Poincaré constant (2.15). Thus, we obtain

$$
\sum_{i=1}^{m(\varepsilon)} \int_{P_{i}^{\ell} \cap \Omega}\left|u_{\varepsilon}\right|^{q} d x \geq C \int_{\Gamma_{1}} \int_{0}^{\varepsilon}\left|u_{\varepsilon}\right|^{2} d x_{N} d S_{x^{\prime}}
$$

Considering the following change of variable $x_{N}=\varepsilon y_{N}$, we have

$$
\int_{\Gamma_{1}} \int_{0}^{\varepsilon}\left|u_{\varepsilon}\right|^{q} d x_{N} d x^{\prime}=\varepsilon \int_{\Gamma_{1}} \int_{0}^{1}\left|u_{\varepsilon}\left(x^{\prime}, \varepsilon y_{N}\right)\right|^{2} d y_{N} d S_{x^{\prime}}
$$

Going back to (3.4), we get by (3.3) that

$$
C \geq \frac{\varepsilon^{b(N-2)}}{\varepsilon^{N-1}} \int_{\Gamma_{1}} \int_{0}^{1}\left|u_{\varepsilon}\left(x^{\prime}, \varepsilon y_{N}\right)\right|^{2} d y_{N} d S_{x^{\prime}}
$$

We pass to the limit as $\varepsilon \rightarrow 0$. Using that $1 \leq b<(N-1) /(N-2)$, we obtain

$$
\lim _{\varepsilon \rightarrow 0} \int_{\Gamma_{1}} \int_{0}^{1}\left|u_{\varepsilon}\left(x^{\prime}, \varepsilon y_{N}\right)\right|^{2} d y_{N} d S_{x^{\prime}}=0 .
$$

Hence, using the regularity of the extremals,

$$
\lim _{\varepsilon \rightarrow 0} \int_{\Gamma_{1}}\left|u_{\varepsilon}\right|^{2} d S=0
$$

Therefore $u_{\varepsilon}$ converges to 0 strongly in $L^{2}\left(\Gamma_{1}\right)$. This shows that every weak limit, $u$, of $u_{\varepsilon}$ in $H^{1}(\Omega)$ verifies $u \equiv 0$ on $\Gamma_{1}$. Therefore

$$
\lambda_{0, \Gamma_{1}} \leq\|u\|_{H^{1}(\Omega)}^{2} \leq \liminf _{\varepsilon \rightarrow 0}\left\|u_{\varepsilon}\right\|_{H^{1}(\Omega)}^{2}=\liminf _{\varepsilon \rightarrow 0} \lambda(\varepsilon) .
$$

From (3.2) and (3.5) we obtain $\lim _{\varepsilon \rightarrow 0} \lambda(\varepsilon)=\lambda_{0, \Gamma_{1}}$. Moreover the above arguments show that the limit $u$ is an extremal of (1.11). This finishes the proof. 


\section{Acknowledgements}

The first and third authors are supported by Fundacion Antorchas, CONICET and ANPCyT PICT 05009 and 10608. The second author was partially supported by Grants S-0505/ESP/0158 of the CAM (Spain) and MTM2005-00714 and MTM200505980 of the MEC.

\section{References}

[1] Adimurthi and S. L. Yadava, "Positive solution for Neumann problem with critical non linearity on boundary", Comm. Partial Differential Equations 16 (1991) 1733-1760.

[2] T. Aubin, "Équations différentielles non linéaires et le problème de Yamabe concernant la courbure scalaire", J. Math. Pures et Appl. 55 (1976) 269-296.

[3] J. Garcia Azorero, I. Peral and J. D. Rossi, "A convex-concave problem with a nonlinear boundary condition", J. Differential Equations 198 (2004) 91-128.

[4] R. J. Biezuner, "Best constants in Sobolev trace inequalities", Nonlinear Analysis 54 (2003) 575589.

[5] J. Fernández Bonder, E. Lami Dozo and J. D. Rossi, "Symmetry properties for the extremals of the Sobolev trace embedding", Ann. Inst. H. Poincaré. Anal. Non Linéaire 21 (2004) 795-805.

[6] J. Fernández Bonder and J. D. Rossi, "On the existence of extremals for the Sobolev trace embedding theorem with critical exponent", Bull. London Math. Soc. 37 (2005) 119-125.

[7] J. Fernández Bonder, J. D. Rossi and N. Wolanski, "Regularity of the free boundary in an optimization problem related to the best Sobolev trace constant", SIAM J. Control Optim. 44 (2005) 1614-1635.

[8] J. Fernández Bonder, J. D. Rossi and N. Wolanski, "Behavior of the best Sobolev trace constant and extremals in domains with holes", Bull. Sci. Math. 130 (2006) 565-579.

[9] P. Cherrier, "Problèmes de Neumann non linéaires sur les variétés Riemanniennes", J. Funct. Anal. 57 (1984) 154-206.

[10] D. Cioranescu and F. Murat, "Un terme étrange venu d'ailleurs", in Nonlinear partial differential equations and their applications. Collège de France Seminar, Vol. II (Paris, 1979/1980), Volume 60 of Res. Notes in Math., English translation: A Strange Term Coming from Nowhere, in Topics in the Mathematical Modelling of Composite Materials, A. Cherkaev et al. eds, Progress in Nonlinear Differential Equations and Their Applications 31, Birkhäuser, Boston (1997) 45-93, (Pitman, Boston, Mass., 1982) 98-138, 389-390.

[11] D. Cioranescu and J. Saint Jean Paulin, "Homogenization in open sets with holes", J. Math. Anal. Appl. 71 (1979) 590-607.

[12] Dö̈na Cioranescu and François Murat, "Un terme étrange venu d'ailleurs. II", in Nonlinear partial differential equations and their applications. College de France Seminar, Vol. III (Paris, 1980/1981), Volume 70 of Res. Notes in Math., (Pitman, Boston, Mass., 1982) 154-178, 425-426.

[13] C. Conca and P. Donato, "Non homogeneous Neumann problems in domains with small holes", RAIRO Modél. Math Anal. Numér. 22 (1988) 561-607.

[14] A. Damlamian and T.-T. Li, "Boundary homogenization for elliptic problems", J. Math. Pures Appl. 66 (1987) 351-361.

[15] O. Druet and E. Hebey, "The $A B$ program in geometric analysis: sharp Sobolev inequalities and related problems", Mem. Amer. Math. Soc. 160 (761) (2002). 
[16] J. F. Escobar, "Sharp constant in a Sobolev trace inequality", Indiana Math. J. 37 (1988) 687-698.

[17] Y. Li and M. Zhu, "Sharp Sobolev trace inequalities on Riemannian manifolds with boundaries", Comm. Pure Appl. Math. 50 (1997) 449-487.

[18] M. Lobo and E. Pérez, "On vibrations of a body with many concentrated masses near the boundary", Math. Models Methods Appl. Sci. 3 (1993) 249-273.

[19] M. Lobo and E. Pérez, "Vibrations of a membrane with many concentrated masses near the boundary", Math. Models Methods Appl. Sci. 5 (1995) 565-585.

[20] F. Murat, "The Neumann sieve", in Nonlinear variational problems (Isola d'Elba, 1983), Volume 127 of Res. Notes in Math., (Pitman, Boston, MA, 1985) 24-32.

[21] J. Rauch and M. Taylor, "Potential and scattering theory on wildly perturbed domains", J. Funct. Anal. 18 (1975) 27-59.

[22] M. W. Steklov, "Sur les problèmes fondamentaux en physique mathématique", Ann. Sci. Ecole Norm. Sup. 19 (1902) 455-490. 